\title{
Impact of Functional Status on Noncardioembolic Ischemic Stroke Recurrence Within 1 Year: The Korean Stroke Cohort for Functioning and Rehabilitation Study
}

\author{
Min-Su Kim ${ }^{a}$, Min Cheol Joo ${ }^{a}$ \\ Min Kyun Sohn ${ }^{\mathrm{b}}$, Jongmin Lee ${ }^{\mathrm{c}}$ \\ Deog Young Kim ${ }^{\mathrm{d}}$, Sam-Gyu Lee \\ Yong-II Shin ${ }^{\dagger}$, Soo-Yeon Kim \\ Gyung-Jae $\mathrm{Oh}^{g}$, Yang-Soo Lee \\ Eun Young Han', Junhee $\mathrm{Han}^{\mathrm{j}}$ \\ Jeonghoon Ahn ${ }^{k}$, Won Hyuk Chang \\ Yun-Hee Kim
}

${ }^{a}$ Department of Rehabilitation Medicine,

Wonkwang University School of Medicine and Institute of Wonkwang Medical Science, Iksan, Korea

Department of Rehabilitation Medicine,

School of Medicine, Chungnam National University, Daejeon, Korea

'Department of Rehabilitation Medicine, Konkuk University School of Medicine, Seoul, Korea

dDepartment and Research Institute of Rehabilitation Medicine, Yonsei University College of Medicine, Seoul, Korea

e Eepartment of Physical and Rehabilitation Medicine, Chonnam National University Medical School, Gwangju, Korea Medical School, Gwangju, Korea 'Department of Rehabilitation Medicine, Pusan National University

Yangsan Hospital, Yangsan, Korea

${ }^{9}$ Department of Preventive Medicine, Wonkwang

University School of Medicine, Iksan, Korea

"Department of Rehabilitation Medicine,

Kyungpook National University School of

Medicine, Kyungpook National University

Medicine, Kyungpook

Hospital, Daegu,

Department of Rehabilitation Medicine, Jeju National University Hospital, Jeju Nationa University School of Medicine, Jeju, Korea Department of Statistics, Hallym University, Chuncheon, Korea

'Department of Health Convergence, Ewha Womans University, Seoul, Korea

'Department of Physical and Rehabilitation

Medicine, Sungkyunkwan University School of

Medicine, Seoul, Korea

Received April 26, 2018

Revised August 20,2018

Accepted August 21, 2018

Correspondence

Yun-Hee Kim, MD, PhD

Department of Physical and

Rehabilitation Medicine,

Sungkyunkwan University

School of Medicine, 81 Irwon-ro,

Gangnam-gu, Seoul 06351, Korea

Tel +82-2-3410-2824

Fax +82-2-3410-0388

E-mail yunkim@skku.edu

Min Cheol Joo, MD, PhD

Department of Rehabilitation Medicine, Wonkwang University School of

Medicine and Institute of Wonkwang

Medical Science, 895 Muwang-ro,

Iksan 54538, Korea

Tel +82-63-859-1610

Fax +82-63-843-1385

E-mail mcjoo68@wku.ac.kr
Background and Purpose Few studies have investigated the relationship between the specific functional factors potentially associated with functional level and stroke recurrence. We conducted a study of patients with noncardioembolic ischemic stroke (NCIS) to determine the functional factors affecting recurrence within the first year.

Methods In total, 568 first-ever NCIS patients (age $=65.1 \pm 17.4$ years, mean \pm SD) were analyzed in a multicenter, prospective cohort study registered from August 2012. Demographic characteristics, past medical history, comorbidities, laboratory data, stroke features in neuroimaging, acute treatments, and medications at discharge were assessed. Functional factors reflecting gross functional impairment, ambulatory function, motor function, activities of daily living, cognition, language ability, swallowing function, mood, and quality of life were comprehensively evaluated in face-to-face assessments using standardized tools at the time of discharge.

Results The cumulative incidence of stroke recurrence in NCIS was $6.0 \%(n=34)$ at 1 year. The period from admission to discharge was $34.4 \pm 7.0$ days. The independent predictors of stroke recurrence within 1 year in multivariate Cox proportional-hazards regression analyses were 1) age [per-year hazard ratio $(\mathrm{HR})=1.04,95 \%$ confidence interval $(\mathrm{CI})=0.97-1.06$, $p=0.048], 2)$ Charlson Comorbidity Index higher than $2(\mathrm{HR}=1.72,95 \% \mathrm{CI}=1.26-2.22, p=$ $0.016), 3$ ) modified Rankin Scale score of 3 or more at discharge (HR=1.56, 95\% CI $=1.22-1.94$, $p=0.032)$, and 4$)$ Functional Ambulation Category of 3 or less at discharge $(\mathrm{HR}=2.56,95 \% \mathrm{CI}=$ $1.84-3.31, p=0.008)$.

Conclusions In addition to patient age, moderate-to-severe functional impairment requiring the help of others (especially for ambulation) at the time of discharge and the severity of comorbidity were independent predictors of stroke recurrence within 1 year of the first NCIS.

Key Words comorbidity, disability evaluation, function, recurrence, stroke.

\section{INTRODUCTION}

Compared to first-ever stroke, recurrent stroke is responsible for greater disability, with moresevere physical, psychological, and social impairments. ${ }^{1}$ Previous community-based studies have revealed that $8-12 \%$ of stroke patients experience recurrence within 1 year after an initial stroke. ${ }^{1-3}$ Risk factors for stroke recurrence include prior stroke, hypertension, heart failure, diabetes, hyperlipidemia, metabolic syndrome, and poor lifestyle, ${ }^{4-7}$ and these factors have been extensively investigated for secondary stroke prevention. Stroke subtype, neuroimaging findings, and several blood markers are also well-recognized risk factors for recurrence, but these factors cannot be modified. ${ }^{8,9}$

While the current approach to secondary prevention primarily focuses on controlling these

(®) This is an Open Access article distributed under the terms of the Creative Commons Attribution Non-Commercial License (https://creativecommons.org/licenses/by-nc/4.0) which permits unrestricted non-commercial use, distribution, and reproduction in any medium, provided the original work is properly cited. 


\section{METHODS}

risk factors, there has been unsatisfactory progress in preventing recurrence. Functional status and rehabilitation are also recently identified factors that are significant for recurrence. ${ }^{10,11}$ Functional status refers to the ability of an individual to perform the normal daily activities required to meet basic needs, fulfill usual roles, and maintain health and well-being. ${ }^{12,13}$ Functional status subsumes related concepts of interest; functional capacity and functional performance. ${ }^{12}$ While functional capacity represents the capacity of an individual to perform daily activities in the physical, psychological, social, and spiritual domains of life, functional performance refers to the activities that people actually participate in during the course of their daily lives. ${ }^{12}$ Functional impairment means the loss of functional status, and it was used in the restricted sense of activity limitation in the present study. ${ }^{14}$ For example, Park and Ovbiagele ${ }^{11}$ reported stepwise relationships between increasing disability and risk of recurrent stroke that contrasted with the outcomes in patients with no disability. Impaired physical activity is a major risk factor for stroke occurrence, and exercise is recommended to prevent stroke and cardiovascular events. ${ }^{15}$ In addition, early functional recovery after stroke can enable survivors to increase their active physical activity and thereby ultimately reduce the probability of stroke recurrence.

However, there has been little research into the potential associations of specific functional indicators with functional impairment and stroke recurrence. It is especially necessary to establish an appropriate rehabilitation strategy to prevent recurrence. Therefore, we conducted a prospective observational study of patients with noncardioembolic ischemic stroke (NCIS) to determine functional indicators that affect recurrence within the first year.

\section{Patient selection}

This study was based on the Korean Stroke Cohort for Functioning and Rehabilitation (KOSCO) report. ${ }^{16}$ The study protocols were approved by the ethics committee of each hospital. The study objectives, design, and risks/benefits were explained to the patients, and written informed consent was obtained upon hospitalization. The study protocols were approved by the ethics committee of each hospital (IRB No. WKUH-1515).

This study enrolled 882 first-ever NCIS patients transferred to a department of rehabilitation after being treated for acute stroke (Fig. 1). Trial of Org 10,172 in Acute Stroke Treatment (TOAST) criteria were used to detect NCIS. ${ }^{17}$ These ischemic stroke subtypes were determined by stroke neurologists at each participating hospital based on neuroimaging findings, medical history, and use of medication. The patients were discharged from the participating hospitals after undergoing inpatient rehabilitation for approximately 3 weeks. Patients who died $(n=19)$ due to causes other than stroke recurrence or were lost to follow-up ( $n=76)$ during the 1-year analysis period were excluded. The causes of death of these patients were as follows: pneumonia $(n=11)$, cardiac event $(n=3)$, cancer $(n=$ $2)$, traffic accident $(n=2)$, and liver cirrhosis $(n=1)$. Among the 787 patients who completed follow-up, hemorrhagic $(n=$ $150)$ and cardioembolic ischemic stroke $(n=69)$ patients were excluded. Therefore, 568 patients with NCIS were finally selected for this study.

\section{Demographic and clinical assessments}

A complete data survey of all patients was conducted based on a review of medical records. Items including demographic data, cerebrovascular risk factors, and family/social history were

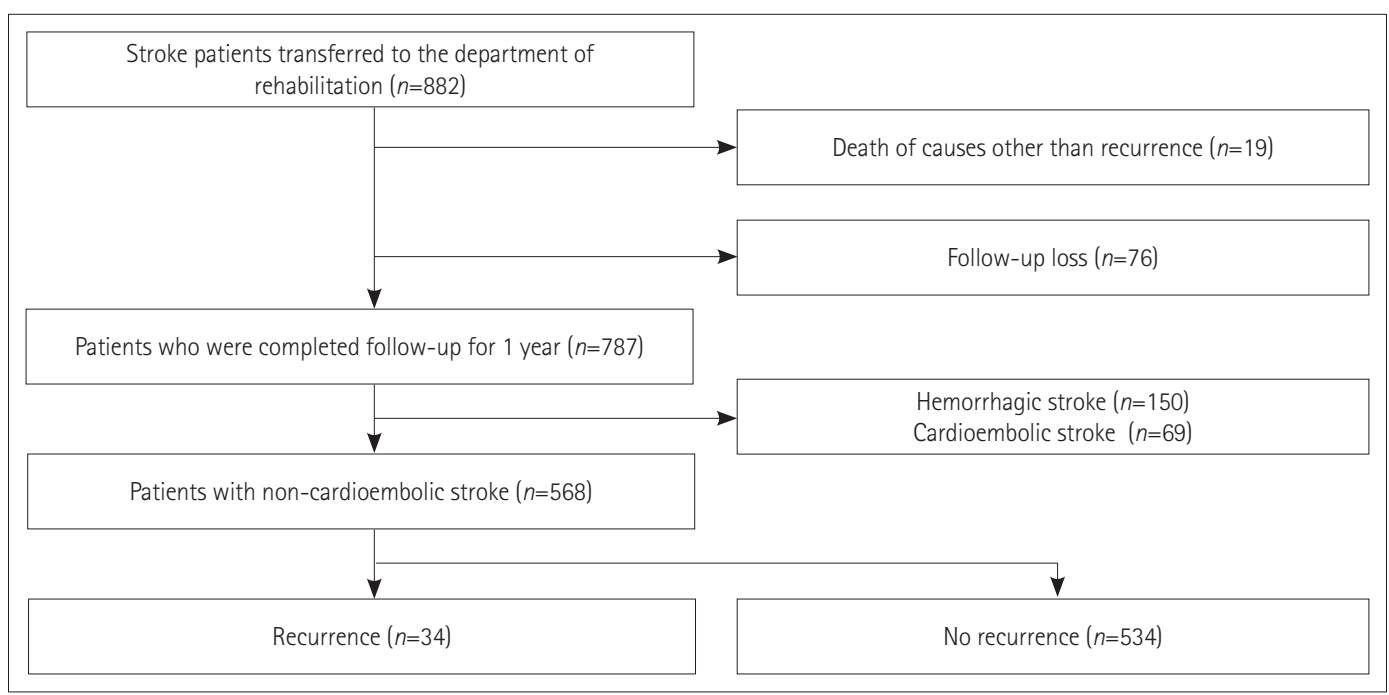

Fig. 1. Patient selection flowchart. 
surveyed using standardized, structured questionnaires. The National Institutes of Health Stroke Scale (NIHSS) was used to evaluate the severity of neurological deficits in the emergency room. Comorbidities were assessed using the Charlson Comorbidity Index (CCI), which contains 19 categories of comorbidity and predicts the mortality of patients who may have a range of comorbid conditions. ${ }^{18-20}$ Because higher CCIs indicate greater comorbidities, we divided the patients into two groups based on a CCI of 2 points, and analyzed the risk of recurrence separately in these groups. ${ }^{18}$ Laboratory data, stroke features, acute treatments, and medications commonly prescribed at discharge were assessed.

\section{Functional status evaluation}

Comprehensive face-to-face functional evaluations were performed at discharge. All evaluations were conducted by research assistants who were sufficiently trained and rigorously tested. The functional status in terms of the level of dependency, ambulation, motor function, activities of daily living, cognition, language ability, swallowing function, mood, and health-related quality of life were evaluated extensively at discharge after receiving stroke rehabilitation using the following standardized tools: modified Rankin Scale (mRS), ${ }^{21}$ Functional Ambulation Category (FAC) ${ }^{22}$ Fugl-Meyer Assessment (FMA) ${ }_{2}^{23}$ Functional Independence Measure (FIM), ${ }^{24}$ Korean version of the Mini Mental State Examination (K-MMSE), ${ }^{25}$ Korean version of the Frenchay Aphasia Screening Test (KFAST), ${ }^{26}$ American Speech-Language-Hearing Association National Outcome Measurement System Swallowing Scale (ASHA-NOMS), ${ }^{27}$ Geriatric Depression Scale-Short Form (GDS-SF), ${ }^{28}$ and EuroQoL-5 Dimensions (EQ-5D), ${ }^{29}$ respectively (Supplementary Table 1 in the online-only Data Supplement).

Several functional indicators were analyzed after dichotomization according to the degree of dependence or severity in order to establish possible rehabilitation strategies in the future. For example, a mRS score of 3 or more indicates that the patient has moderate-to-severe functional impairment, and a FAC score of 3 or less means that the patient requires manual contact with another person during ambulation on level surfaces. The FMA motor score was divided into two groups according to the severity: mild (>79) and moderate to severe $(0-79){ }^{23}$ The FIM ranges from 18 to 126 points and is a common tool used for assessing the ability to perform the activities of daily living. ${ }^{24}$ These assessments were performed in the present study based on dividing the level of dependence on others into two groups: mild $(>90)$ and moderate to severe $(18-90){ }^{24}$ The K-MMSE score was analyzed by dividing the patients into two groups, with scores of $0-23$ points indicating moderate-to-severe cognitive impairment and scores of more than 23 points indicating mild cognitive dysfunction. ${ }^{25}$ The ASHA-NOMS scores were also analyzed by dividing patients into two groups corresponding to normal (7 points) and abnormal ( $\leq 6$ points) swallowing function.

\section{Identification of stroke recurrence within 1 year}

Detailed information about stroke recurrence was collected at 3 months, 6 months, and 1 year after stroke onset. All patients were approached for follow-up assessments using a twostep approach. ${ }^{16}$ We first regularly obtained follow-up information via telephone interviews with the patients themselves or their caregivers. All of the survivors were subsequently invited to visit the outpatient clinics of the hospitals where they were first admitted to complete a battery of functional assessments, structured self-administered questionnaires, and faceto-face interviews. If patients were not able to return for the follow-up assessments, research assistants visited the patients at their homes to perform these same evaluations. Stroke recurrence was confirmed based on the medical records of each participating hospital whenever the patients themselves or their caregivers reported recurrence during these follow-up initiatives.

\section{Statistical analysis}

We used univariate Cox proportional-hazards regression to identify differences in baseline characteristics between patients with and without recurrence. Hazard ratios (HRs) and 95\% confidence intervals (CIs) were calculated using the univariate Cox proportional-hazards model. We also determined the risk of subsequent stroke recurrence in relation to each variable by using multivariate Cox proportional-hazards regression analysis. A backward conditional procedure was performed using $p>0.10$ in the likelihood ratio test for exclusion. The regression model included the time to recurrent stroke as the response, and clinical/functional predictors of recurrence with a univariate probability value of $p<0.1$ as an independent variable. Because sex and the initial NIHSS score measured in the emergency room are well-known factors involved in stroke recurrence, ${ }^{30-32}$ these factors were additionally included and analyzed in the final model. The Kaplan-Meier method was used to evaluate the distribution of time to events according to the characteristics derived from the multivariate Cox proportional-hazards regression analysis. Intergroup comparisons were performed using the log-rank test. A probability value of $p<0.05$ was considered significant. All analyses were performed using SPSS statistical software (version 22.0; IBM Corp., Armonk, NY, USA). 
Table 1. Clinical characteristics of the patients and HRs from the univariate Cox proportional-hazards regression analyses for stroke recurrence

\begin{tabular}{|c|c|c|c|c|}
\hline & Recurrence: yes $(n=34, \%)$ & Recurrence: no $(n=534, \%)$ & HR $(95 \% \mathrm{Cl})$ & $p$ \\
\hline \multicolumn{5}{|l|}{ Baseline characteristics } \\
\hline Age, years & $70.3 \pm 13.0$ & $63.7 \pm 19.4$ & $1.04(0.97-1.06)$ & $0.016^{*}$ \\
\hline Sex, male & 60 & 58 & $1.21(0.67-1.82)$ & 0.309 \\
\hline $\mathrm{BMI}, \mathrm{kg} / \mathrm{m}^{2}$ & $23.1 \pm 3.1$ & $23.5 \pm 3.4$ & $0.96(0.90-1.02)$ & 0.209 \\
\hline Marital status, married & 79 & 78 & $1.17(0.45-2.02)$ & 0.470 \\
\hline Education, $\geq$ high school & 48 & 49 & $1.00(0.38-1.62)$ & 0.992 \\
\hline Family history & 11 & 9 & $1.48(0.98-2.12)$ & 0.169 \\
\hline Smoking & 57 & 53 & $1.06(0.82-1.35)$ & 0.771 \\
\hline Alcohol & 65 & 62 & $1.11(0.74-1.55)$ & 0.582 \\
\hline $\mathrm{CCl}_{1}>2$ & 27 & 15 & $2.20(1.30-3.24)$ & $0.008^{*}$ \\
\hline \multicolumn{5}{|l|}{ Past medical history } \\
\hline Hypertension & 61 & 58 & $1.05(0.46-1.67)$ & 0.781 \\
\hline Diabetes mellitus & 30 & 29 & $1.34(0.40-2.33)$ & 0.134 \\
\hline Ischemic heart disease & 8 & 7 & $1.20(0.52-1.73)$ & 0.596 \\
\hline Atrial fibrillation & 8 & 6 & $1.80(1.02-3.03)$ & $0.060^{*}$ \\
\hline Hyperlipidemia & 13 & 10 & $1.73(1.12-2.88)$ & $0.055^{*}$ \\
\hline \multicolumn{5}{|c|}{ Initial neurological deficits in the emergency room } \\
\hline NIHSS score & $8.6 \pm 6.2$ & $9.5 \pm 6.9$ & $1.01(0.74-1.52)$ & 0.325 \\
\hline \multicolumn{5}{|l|}{ Laboratory data on admission } \\
\hline Fasting glucose, mg/dL & $101 \pm 16$ & $104 \pm 18$ & $1.00(0.89-1.11)$ & 0.681 \\
\hline Total cholesterol, mg/dL & $176 \pm 41$ & $178 \pm 44$ & $0.99(0.95-1.05)$ & 0.325 \\
\hline $\mathrm{LDL}-\mathrm{C}, \mathrm{mg} / \mathrm{dL}$ & $105 \pm 32$ & $108 \pm 36$ & $1.00(0.89-1.09)$ & 0.483 \\
\hline $\mathrm{HDL}-\mathrm{C}, \mathrm{mg} / \mathrm{dL}$ & $45 \pm 13$ & $46 \pm 17$ & $0.98(0.90-1.06)$ & 0.889 \\
\hline Triglycerides, mg/dL & $124 \pm 76$ & $121 \pm 78$ & $1.00(0.88-1.12)$ & 0.450 \\
\hline Creatinine, mg/dL & $0.9 \pm 0.6$ & $1.0 \pm 0.6$ & $0.94(0.90-1.00)$ & 0.699 \\
\hline Homocysteine, $\mu \mathrm{mol} / \mathrm{L}$ & $10.7 \pm 17.5$ & $9.2 \pm 10.6$ & $1.01(0.86-1.16)$ & 0.812 \\
\hline \multicolumn{5}{|l|}{ Stroke features } \\
\hline Lesion side, left & 50 & 46 & $0.83(0.39-1.69)$ & 0.296 \\
\hline Location, brain stem & 30 & 30 & $1.07(0.68-1.52)$ & 0.729 \\
\hline \multicolumn{5}{|l|}{ Subtypes } \\
\hline LAA & 81 & 77 & $2.19(1.33-3.42)$ & $0.028^{*}$ \\
\hline Small-vessel occlusion & 18 & 21 & $1.02(0.60-1.45)$ & 0.113 \\
\hline Others & 1 & 2 & & \\
\hline \multicolumn{5}{|l|}{ Acute-phase therapies } \\
\hline IV tPA & 5 & 6 & $0.85(0.49-1.16)$ & 0.712 \\
\hline IA tPA & 5 & 4 & $1.46(0.68-2.32)$ & 0.224 \\
\hline IV heparin & 9 & 10 & $1.18(0.63-1.94)$ & 0.328 \\
\hline Antiplatelet & 81 & 80 & $1.44(1.02-1.82)$ & 0.123 \\
\hline \multicolumn{5}{|l|}{ Medications at discharge } \\
\hline Antiplatelet & 71 & 76 & $0.84(0.45-1.26)$ & 0.443 \\
\hline Anticoagulation & 10 & 10 & $0.99(0.82-1.19)$ & 0.220 \\
\hline Antihypertensive & 47 & 41 & $1.26(0.94-1.66)$ & 0.199 \\
\hline Antidiabetic & 24 & 19 & $1.08(0.79-1.28)$ & 0.748 \\
\hline Antilipid & 73 & 68 & $1.20(0.93-1.63)$ & 0.347 \\
\hline
\end{tabular}

Noncategoric data are expressed as mean \pm SD values. Categoric data are expressed as percentage values.

${ }^{*} p<0.10$.

BMI: body mass index, CCl: Charlson Comorbidity Index, Cl: confidence interval, HDL-C: high-density lipoprotein cholesterol, HR: hazard ratio, IA: intraarterial, IV: intravenous, LAA: large-artery atherosclerosis, LDL-C: low-density lipoprotein cholesterol, NIHSS: National Institutes of Health Stroke Scale, tPA: tissue plasminogen activator. 


\section{RESULTS}

This study analyzed 568 patients (age $=65.1 \pm 17.4$ years, mean \pm SD; 318 males and 250 females), of which 34 patients experienced recurrent stroke during the 1-year observation period, giving a recurrence rate following NCIS of $6.0 \%$ (34/568). Four patients died due to stroke recurrence. The period to discharge after stroke onset was $34.4 \pm 7.0$ days, and we identified that NCIS was caused by large-artery atherosclerosis in 446 (79\%) patients, small-vessel occlusion in 108 (19\%) patients, and another cause in 14 (2\%) patients.

\section{Univariate analyses}

The clinical characteristics, laboratory data, stroke features, and medications prescribed at discharge are presented in Table 1. Age $(\mathrm{HR}=1.04,95 \% \mathrm{CI}=0.97-1.06, p=0.016)$, a CCI higher than $2(\mathrm{HR}=2.20,95 \% \mathrm{CI}=1.30-3.24, p=0.008)$, atrial fibrillation $(\mathrm{HR}=1.80,95 \% \mathrm{CI}=1.02-3.03, p=0.060)$, hyperlipidemia $(\mathrm{HR}=1.73,95 \% \mathrm{CI}=1.12-2.88, p=0.055)$, and large-artery atherosclerosis $(\mathrm{HR}=2.19,95 \% \mathrm{CI}=1.33-3.42, p=0.028)$ were significantly associated with stroke recurrence.

Table 2 presents the results for the functional factors associated with the risk of stroke recurrence. The period to discharge after stroke onset was $34.8 \pm 6.8$ days in the patients with recurrent stroke and $34.2 \pm 7.0$ days in those who were recurrence-free during the 1-year analysis period. Significant associations with the risk of stroke recurrence were found for an $\mathrm{mRS}$ score of 3 or more $(\mathrm{HR}=1.85,95 \% \mathrm{CI}=1.28-2.54, p=$ $0.010)$, an FAC score of 3 or less ( $\mathrm{HR}=3.89,95 \% \mathrm{CI}=2.32-5.65$, $p<0.001)$, and an FMA motor score of 79 or less $(\mathrm{HR}=1.46$, $95 \% \mathrm{CI}=1.02-1.90, p=0.048)$.

\section{Multivariate analyses}

A subsequent analysis was performed of all significant variables after the univariate analyses. As indicated in Table 3, the independent predictors of stroke recurrence within 1 year after onset in the multivariate Cox proportional-hazards regression analysis were 1 ) patient age (per-year $\mathrm{HR}=1.04$, 95\% CI=0.97-1.06, $p=0.048), 2) \mathrm{CCI}$ higher than $2(\mathrm{HR}=$ $1.72,95 \% \mathrm{CI}=1.26-2.22, p=0.016), 3) \mathrm{mRS}$ score of 3 or more

Table 2. Functional status assessed at discharge and the results of the univariate Cox proportional-hazards regression analysis

\begin{tabular}{|c|c|c|c|c|}
\hline Functional indicator & Recurrence: yes $(n=34, \%)$ & Recurrence: no $(n=534, \%)$ & $\mathrm{HR}(95 \% \mathrm{Cl})$ & $p$ \\
\hline$m R S$ score,$\geq 3$ & 42 & 35 & $1.85(1.28-2.54)$ & $0.010^{*}$ \\
\hline FAC score, $\leq 3$ & 47 & 34 & $3.89(2.32-5.65)$ & $<0.001^{*}$ \\
\hline FMA motor score, 0-79 & 48 & 42 & $1.46(1.02-1.90)$ & $0.048^{*}$ \\
\hline FIM, 18-90 & 50 & 46 & $1.34(0.94-1.74)$ & 0.126 \\
\hline K-MMSE score, 0-23 & 49 & 51 & $1.12(0.86-1.40)$ & 0.233 \\
\hline K-FAST score & $6.1 \pm 1.7$ & $6.2 \pm 1.6$ & $1.04(0.80-1.28)$ & 0.421 \\
\hline ASHA-NOMS score, $\leq 6$ & 32 & 29 & $0.78(0.29-1.33)$ & 0.250 \\
\hline GDS-SF score & $2.6 \pm 1.6$ & $2.2 \pm 1.5$ & $0.99(0.80-1.19)$ & 0.407 \\
\hline EQ-5D score & $0.65 \pm 0.37$ & $0.60 \pm 0.35$ & $1.01(0.98-1.04)$ & 0.207 \\
\hline Period to discharge, days & $34.8 \pm 6.8$ & $34.2 \pm 7.0$ & $0.93(0.84-1.04)$ & 0.124 \\
\hline
\end{tabular}

Noncategoric data are expressed as mean \pm SD values. Categoric data are expressed as percentage values.

${ }^{*} p<0.10$.

ASHA-NOMS: American Speech-Language-Hearing Association National Outcome Measurement System Swallowing Scale, Cl: confidence interval, EQ-5D: EuroQoL-5 Dimensions, FAC: Functional Ambulation Category, FIM: Functional Independence Measure, FMA: Fugl-Meyer Assessment, GDS-SF: Geriatric Depression Scale-Short Form, HR: hazard ratio, K-FAST: Korean version of the Frenchay Aphasia Screening Test, K-MMSE: Korean version of the Mini Mental State Examination, mRS: modified Rankin Scale.

Table 3. Results of multivariate Cox proportional-hazards regression analyses for stroke recurrence

\begin{tabular}{|c|c|c|c|}
\hline & HR & $95 \% \mathrm{Cl}$ & $p$ \\
\hline Age & 1.04 & $0.97-1.06$ & $0.048^{*}$ \\
\hline Sex, male & 1.08 & $0.87-1.30$ & 0.374 \\
\hline NIHSS score in the emergency room & 1.01 & $0.84-1.18$ & 0.538 \\
\hline $\mathrm{CCl}_{1}>2$ & 1.72 & $1.26-2.22$ & $0.016^{*}$ \\
\hline LAA & 1.23 & $1.06-1.59$ & 0.082 \\
\hline $\mathrm{mRS}$ score at discharge, $\geq 3$ & 1.56 & $1.22-1.94$ & $0.032^{*}$ \\
\hline FAC score at discharge,$\leq 3$ & 2.56 & $1.84-3.31$ & $0.008^{*}$ \\
\hline
\end{tabular}

${ }^{*} p<0.05$.

CCI: Charlson Comorbidity Index, Cl: confidence interval, FAC: Functional Ambulation Category, HR: hazard ratio, LAA: large-artery atherosclerosis, mRS: modified Rankin Scale, NIHSS: National Institutes of Health Stroke Scale. 

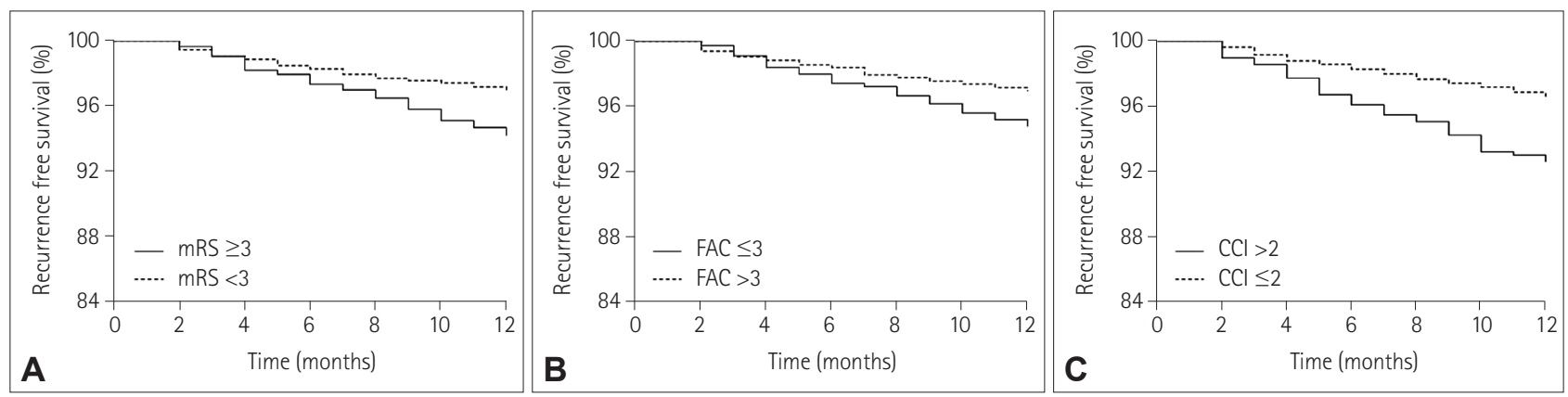

Fig. 2. Differences in the recurrence-free survival according to the Kaplan-Meier analysis. Significant differences in recurrence-free survival rate were found for the mRS score (A), the FAC score at discharge (B), and the CCI (C). CCl: Charlson Comorbidity Index, FAC: Functional Ambulatory Category, mRS: modified Rankin Scale.

(HR=1.56, 95\% CI=1.22-1.94, $p=0.032)$, and 4) FAC score of 3 or less ( $\mathrm{HR}=2.56,95 \% \mathrm{CI}=1.84-3.31, p=0.008)$. According to the Kaplan-Meier analysis, there were significant differences in recurrence-free survival between patients with $\mathrm{mRS}$ score $\geq 3$ and $\mathrm{mRS}$ score $<3$ at discharge ( $p=0.034$, log-rank test), and between patients with FAC score $\leq 3$ and FAC score $>3$ at discharge ( $p=0.016$, log-rank test) (Fig. 2). In addition, the recurrence-free survival period was significantly shorter for patients with CCI $>2$ than for those with CCI $\leq 2$ ( $p=0.028$, log-rank test).

\section{DISCUSSION}

The present study found that functional impairment (especially ambulatory function) at discharge, age, and the severity of comorbidities were independent predictors of recurrence within 1 year after the onset of NCIS. The results of this study imply that applying other interventions to promote functionin addition to previously developed medical treatments for secondary stroke prevention-may reduce the probability of stroke recurrence.

The functional status based on the mRS score after simplification into two categories (no or slight functional impairment and moderate-to-severe functional impairment) was a strong indicator of recurrence. It was previously shown that functional impairment at approximately 1 month poststroke is related to long-term functional outcome, ${ }^{21}$ and the present results are similar to those reported previously. ${ }^{11,33}$ It was reported that compared to no/slight functional impairment (mRS score $\leq 2)$, moderate functional impairment ( $\mathrm{mRS}$ score $=3$ ) after a recent NCIS was independently associated with an almost 1.5 -fold higher risk of recurrent stroke within 2 years. ${ }^{11}$ The mRS did not indicate impairment of any specific functional domains, since it is an index of the overall dependence according to the severity of gross functional impairment. ${ }^{34}$ However, the previous study did not precisely consider what functional deficits were underlying these findings. Instead, reactive depression arising from clearly more-disabling consequences, and vitamin D deficiency due to limited mobility and decreased sunlight exposure were thought to be the factors underlying the low mRS scores and stroke recurrence. ${ }^{11}$

In contrast, our present comprehensive analysis of overall functional indicators has confirmed that ambulatory function is a critical factor for stroke recurrence. Those patients who needed help from others or were not able to walk at all (FAC score $\leq 3$ ) were approximately 2.5 -fold more likely to experience recurrence than those who did not have such deficits. The underlying mechanisms linking ambulatory function and stroke recurrence remain unclear, but they can be inferred from several reports. Kono et al. ${ }^{35}$ reported that physical activity quantified based on the step count during ambulation was an independent predictor of the recurrence of vascular events (68\% for stroke and 32\% for coronary heart disease) in stroke survivors. It is widely known that lifestyle modifications for secondary stroke prevention significantly reduce the probability of stroke recurrence and other vascular events, and physical activity also tends to be associated with stroke recurrence. ${ }^{73,37}$ Another study found that walking itself enhanced endogenous fibrinolysis by increasing tissue plasminogen activator and decreasing the activity of plasminogen activator inhibitor PAI-1 in stroke patients. ${ }^{38}$ Moreover, Cheng et al. ${ }^{10}$ discovered that rehabilitation during the first year after stroke was significantly associated with a lower incidence of recurrent stroke and all-cause mortality. Therefore, a rehabilitation strategy that focuses on the recovery of ambulatory function should be strongly considered to prevent recurrence.

Comorbidity was also a strong predisposing factor for recurrence in patients with NCIS. Although there has been little research into the relevance of comorbidities to stroke recurrence, a few possible mechanisms can be considered. It is known that the CCI is a strong predictor of both mortality and stroke occurrence. ${ }^{18,39}$ A previous comparison of very severe comorbidity with no comorbidity found ischemic stroke increased both the 30 -day and 5-year mortality rates by approx- 
imately 2.5 -fold. ${ }^{18}$ Comorbidity is also an important factor in poststroke functional recovery. ${ }^{40}$ Insufficient rehabilitation therapies and repeated hospitalization due to multiple comorbidities contribute to poor functional outcomes. ${ }^{41}$ Poor functional recovery in patients with multiple comorbidities cause decreased physical activity and deconditioning, ${ }^{42,43}$ which lead to arterial stiffness, increased atherosclerosis, and failure to control other stroke risk factors. ${ }^{43,44}$ These processes were therefore considered to increase the probability of stroke recurrence.

Several limitations of the present study should be considered when interpreting its findings. We confirmed stroke recurrence after calling the patients themselves or their caregivers by telephone at each time point. Although the research assistants visited the homes of the patients after establishing phone contact, a limitation was the difficulty of determining recurrence if the patients or caregivers could not be reached by telephone. Furthermore, a larger number of recurrent stroke patients may be missed by our protocol compared with a methodology utilizing a nationwide stroke registry to identify recurrence. The functional data at discharge of the patients who were not followed up are summarized in Supplementary Table 1 (in the online-only Data Supplement). We thought that patients with greater functional impairment due to neurological deficits were more likely to be lost to follow-up, but there were no differences in functional status. We consider it most likely that these patients stopped participating in the research intentionally during the follow-up for personal reasons. The degree of compliance with the protocol of this study was not investigated, and so the factors influencing compliance should be analyzed in a future study. Although the 76 patients who were lost to follow-up within 1 year accounted for a small proportion of the overall population, their exclusion may have influenced the results. In addition, information on adherence to medications for secondary prevention and good health-related behaviors was not collected during the follow-up. In Korea, medication prescriptions for secondary prevention and educating about health-related behaviors are mainly the responsibility of neurologists. However, the researchers visiting the present patients received regular training about medications and habitual education. They also taught the patients to comply with taking their medications and follow their instructions for behavioral changes during every visit. However, the actual compliance with these activities was not investigated in this study.

In conclusion, moderate-to-severe functional impairment at the time of discharge requiring the help of others (especially for ambulation) and the severity of comorbidity in addition to age were confirmed to be independent risk factors for stroke recurrence within 1 year of onset in patients with
NCIS. Additional treatments focused on the recovery of ambulatory function may be a good strategy for preventing stroke recurrence.

\section{Supplementary Materials}

The online-only Data Supplement is available with this article at https://doi.org/10.3988/jcn.2019.15.1.54.

\section{Conflicts of Interest}

The authors have no financial conflicts of interest.

\section{Acknowledgements}

This study was supported by the Research Program funded by the Korea Centers for Disease Control and Prevention (2016E-33003-02), Korea.

\section{REFERENCES}

1. Elkind MS. Outcomes after stroke: risk of recurrent ischemic stroke and other events. Am J Med 2009;122(4 Suppl 2):S7-S13.

2. Kuwashiro T, Sugimori H, Ago T, Kamouchi M, Kitazono T; FSR Investigators. Risk factors predisposing to stroke recurrence within one year of non-cardioembolic stroke onset: the Fukuoka Stroke Registry. Cerebrovasc Dis 2012;33:141-149.

3. Hillen T, Coshall C, Tilling K, Rudd AG, McGovern R, Wolfe CD; South London Stroke Register. Cause of stroke recurrence is multifactorial: patterns, risk factors, and outcomes of stroke recurrence in the south London stroke register. Stroke 2003;34:1457-1463.

4. Callaly E, Ni Chroinin D, Hannon N, Marnane M, Akijian L, Sheehan $\mathrm{O}$, et al. Rates, predictors, and outcomes of early and late recurrence after stroke: the North Dublin Population Stroke Study. Stroke 2016;47:244-246.

5. Andersen SD, Gorst-Rasmussen A, Lip GY, Bach FW, Larsen TB. Recurrent stroke: the value of the CHA2DS2VASc score and the essen stroke risk score in a nationwide stroke cohort. Stroke 2015;46:24912497.

6. Furie KL, Kasner SE, Adams RJ, Albers GW, Bush RL, Fagan SC, et al. Guidelines for the prevention of stroke in patients with stroke or transient ischemic attack: a guideline for healthcare professionals from the American Heart Association/American Stroke Association. Stroke 2011;42:227-276.

7. Kono Y, Yamada S, Kamisaka K, Araki A, Fujioka Y, Yasui K, et al. Recurrence risk after noncardioembolic mild ischemic stroke in a Japanese population. Cerebrovasc Dis 2011;31:365-372.

8. Elkind MS, Luna JM, McClure LA, Zhang Y, Coffey CS, Roldan A, et al. C-reactive protein as a prognostic marker after lacunar stroke: levels of inflammatory markers in the treatment of stroke study. Stroke 2014;45:707-716.

9. Kim GM, Park KY, Avery R, Helenius J, Rost N, Rosand J, et al. Extensive leukoaraiosis is associated with high early risk of recurrence after ischemic stroke. Stroke 2014;45:479-485.

10. Cheng YY, Shu JH, Hsu HC, Liang Y, Chang ST, Kao CL, et al. The impact of rehabilitation frequencies in the first year after stroke on the risk of recurrent stroke and mortality. J Stroke Cerebrovasc Dis 2017; 26:2755-2762.

11. Park JH, Ovbiagele B. Relationship of functional disability after a recent stroke with recurrent stroke risk. Eur J Neurol 2016;23:361-367.

12. Leidy NK. Functional status and the forward progress of merry-gorounds: toward a coherent analytical framework. Nurs Res 1994;43: 196-202.

13. Wilson IB, Cleary PD. Linking clinical variables with health-related quality of life. A conceptual model of patient outcomes. JAMA 1995; 273:59-65. 
14. Ustün B, Kennedy C. What is "functional impairment?" Disentangling disability from clinical significance. World Psychiatry 2009;8: 82-85.

15. Saunders DH, Greig CA, Mead GE. Physical activity and exercise after stroke: review of multiple meaningful benefits. Stroke 2014;45:37423747.

16. Chang WH, Sohn MK, Lee J, Kim DY, Lee SG, Shin YI, et al. Korean stroke cohort for functioning and rehabilitation (KOSCO): study rationale and protocol of a multi-centre prospective cohort study. BMC Neurol 2015;15:42.

17. Adams HP Jr, Bendixen BH, Kappelle LJ, Biller J, Love BB, Gordon $\mathrm{DL}$, et al. Classification of subtype of acute ischemic stroke. Definitions for use in a multicenter clinical trial. TOAST. Trial of Org 10172 in acute stroke treatment. Stroke 1993;24:35-41.

18. Schmidt M, Jacobsen JB, Johnsen SP, Bøtker HE, Sørensen HT. Eighteen-year trends in stroke mortality and the prognostic influence of comorbidity. Neurology 2014;82:340-350.

19. Chang WH, Sohn MK, Lee J, Kim DY, Lee SG, Shin YI, et al. Predictors of functional level and quality of life at 6 months after a first-ever stroke: the KOSCO study. J Neurol 2016;263:1166-1177.

20. Fried L, Bernardini J, Piraino B. Charlson comorbidity index as a predictor of outcomes in incident peritoneal dialysis patients. Am J Kidney Dis 2001;37:337-342.

21. Ovbiagele B, Lyden PD, Saver JL; VISTA Collaborators. Disability status at 1 month is a reliable proxy for final ischemic stroke outcome. Neurology 2010;75:688-692.

22. Mehrholz J, Wagner K, Rutte K, Meissner D, Pohl M. Predictive validity and responsiveness of the functional ambulation category in hemiparetic patients after stroke. Arch Phys Med Rehabil 2007;88:13141319.

23. Duncan PW, Goldstein LB, Horner RD, Landsman PB, Samsa GP, Matchar DB. Similar motor recovery of upper and lower extremities after stroke. Stroke 1994;25:1181-1188.

24. Ancheta J, Husband M, Law D, Reding M. Initial functional independence measure score and interval post stroke help assess outcome, length of hospitalization, and quality of care. Neurorehabil Neural Repair 2000;14:127-134.

25. Mungas D. In-office mental status testing: a practical guide. Geriatrics 1991;46:54-58, 63, 66.

26. Enderby P, Crow E. Frenchay aphasia screening test: validity and comparability. Disabil Rehabil 1996;18:238-240.

27. Kim J, Oh BM, Lee GJ, Lee SA, Chun SW, Han TR. Clinical factors associated with severity of post-stroke dysphagia. Brain Neurorehabil 2011;4:116-120.

28. Roger PR, Johnson-Greene D. Comparison of assessment measures for post-stroke depression. Clin Neuropsychol 2009;23:780-793.

29. Golicki D, Niewada M, Buczek J, Karlińska A, Kobayashi A, Janssen MF, et al. Validity of EQ-5D-5L in stroke. Qual Life Res 2015;24:845850 .

30. Toni D, Di Angelantonio E, Di Mascio MT, Vinisko R, Bath PM; PRo-
FESS Study Group. Types of stroke recurrence in patients with ischemic stroke: a substudy from the PRoFESS trial. Int J Stroke 2014;9: 873-878.

31. Omori T, Kawagoe M, Moriyama M, Yasuda T, Ito Y, Hyakuta T, et al. Multifactorial analysis of factors affecting recurrence of stroke in Japan. Asia Pac J Public Health 2015;27:NP333-NP340.

32. Zhang C, Zhao X, Wang C, Liu L, Ding Y, Akbary F, et al. Prediction factors of recurrent ischemic events in one year after minor stroke. PLoS One 2015;10:e0120105.

33. Magalhães R, Abreu P, Correia M, Whiteley W, Silva MC, Sandercock P. Functional status three months after the first ischemic stroke is associated with long-term outcome: data from a community-based cohort. Cerebrovasc Dis 2014;38:46-54.

34. de Haan R, Limburg M, Bossuyt P, van der Meulen J, Aaronson N. The clinical meaning of Rankin 'handicap' grades after stroke. Stroke 1995;26:2027-2030.

35. Kono Y, Kawajiri H, Kamisaka K, Kamiya K, Akao K, Asai C, et al. Predictive impact of daily physical activity on new vascular events in patients with mild ischemic stroke. Int J Stroke 2015;10:219-223.

36. Ovbiagele B, Saver JL, Fredieu A, Suzuki S, Selco S, Rajajee V, et al. In-hospital initiation of secondary stroke prevention therapies yields high rates of adherence at follow-up. Stroke 2004;35:2879-2883.

37. Kono Y, Yamada S, Yamaguchi J, Hagiwara Y, Iritani N, Ishida S, et al. Secondary prevention of new vascular events with lifestyle intervention in patients with noncardioembolic mild ischemic stroke: a singlecenter randomized controlled trial. Cerebrovasc Dis 2013;36:88-97.

38. Ivey FM, Womack CJ, Kulaputana O, Dobrovolny CL, Wiley LA, Macko RF. A single bout of walking exercise enhances endogenous fibrinolysis in stroke patients. Med Sci Sports Exerc 2003;35:193-198.

39. Tang J, Wan JY, Bailey JE. Performance of comorbidity measures to predict stroke and death in a community-dwelling, hypertensive Medicaid population. Stroke 2008;39:1938-1944.

40. Tessier A, Finch L, Daskalopoulou SS, Mayo NE. Validation of the Charlson comorbidity index for predicting functional outcome of stroke. Arch Phys Med Rehabil 2008;89:1276-1283.

41. Yeh HJ, Huang N, Chou YJ, Cheng SP, Lee WK, Lai CC, et al. Older age, low socioeconomic status, and multiple comorbidities lower the probability of receiving inpatient rehabilitation half a year after stroke. Arch Phys Med Rehabil 2017;98:707-715.

42. Kyu HH, Bachman VF, Alexander LT, Mumford JE, Afshin A, Estep K, et al. Physical activity and risk of breast cancer, colon cancer, diabetes, ischemic heart disease, and ischemic stroke events: systematic review and dose-response meta-analysis for the Global Burden of Disease Study 2013. BMJ 2016;354:i3857.

43. Howard VJ, McDonnell MN. Physical activity in primary stroke prevention: just do it! Stroke 2015;46:1735-1739.

44. Tang A, Eng JJ, Brasher PM, Madden KM, Mohammadi A, Krassioukov AV, et al. Physical activity correlates with arterial stiffness in community-dwelling individuals with stroke. J Stroke Cerebrovasc Dis 2014;23:259-266. 